\title{
Diet-Right: A Smart Food Recommendation System
}

\author{
Faisal Rehman ${ }^{1, *}$, Osman Khalid ${ }^{1}$, Nuhman ul Haq ${ }^{1}$, Atta ur Rehman Khan ${ }^{2}$, Kashif Bilal, \\ and Sajjad A. Madani ${ }^{1}$ \\ ${ }^{1}$ Department of Computer Sciences, COMSATS Institute of Information Technology \\ Abbottabad, Pakistan \\ [e-mail: frehman, osman, nuhman, kashifbilal, madani @ciit.net.pk] \\ ${ }^{2}$ College of Computer \& Information Sciences, King Saud University \\ Riyadh, Saudi Arabia \\ [e-mail: dr@attaurrehman.com] \\ *Corresponding author: Faisal Rehman
}

Received October 21, 2016; revised January 2, 2017; accepted February 14, 2017;

published June 30, 2017

\begin{abstract}
Inadequate and inappropriate intake of food is known to cause various health issues and diseases. Due to lack of concise information about healthy diet, people have to rely on medicines instead of taking preventive measures in food intake. Due to diversity in food components and large number of dietary sources, it is challenging to perform real-time selection of diet patterns that must fulfill one's nutrition needs. Particularly, selection of proper diet is critical for patients suffering from various diseases. In this article, we highlight the issue of selection of proper diet that must fulfill patients' nutrition requirements. To address this issue, we present a cloud based food recommendation system, called Diet-Right, for dietary recommendations based on users' pathological reports. The model uses ant colony algorithm to generate optimal food list and recommends suitable foods according to the values of pathological reports. Diet-Right can play a vital role in controlling various diseases. The experimental results show that compared to single node execution, the convergence time of parallel execution on cloud is approximately 12 times lower. Moreover, adequate accuracy is attainable by increasing the number of ants.
\end{abstract}

Keywords: Recommender System, Food, eHealth, ACO, Cloud Computing, Pathological Reports

A preliminary version of this paper appeared in IEEE ICC 2009, June 14-18, Dresden, Germany. This version includes a concrete analysis and supporting implementation results on MICAz sensor nodes. This research was supported by a research grant from the IT R\&D program of MKE/IITA, the Korean government [2005-Y-001-04, Development of Next Generation Security Technology]. We express our thanks to Dr. Richard Berke who checked our manuscript. 


\section{Introduction}

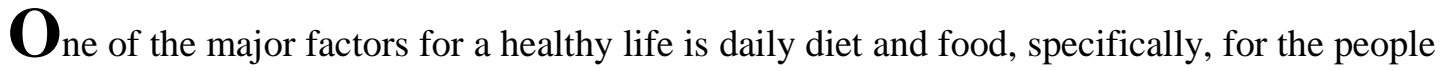
suffering from some minor or major diseases. eHealth initiatives and research efforts aim to offer various pervasive applications for novice end users to improve their health [1]. Various studies depict that inappropriate and inadequate intake of daily diet are the major reasons of various health issues and diseases. A study conducted by World Health Organization (WHO) estimates that around $30 \%$ of the total population of the world is suffering from various diseases, and $60 \%$ deaths each year in children are related to malnutrition [2, 3]. Another study by WHO reports that inadequate and imbalanced intake of food causes around $9 \%$ of heart attack deaths, about $11 \%$ of ischemic heart disease deaths, and $14 \%$ of gastrointestinal cancer deaths worldwide [4]. Moreover, around 0.25 billion children are suffering from Vitamin-A deficiency [5], 0.2 billion people are suffering from iron deficiency (anemia) [6], and 0.7 billion people are suffering from iodine deficiency [7].

Generally, a person remains unaware of major causes behind deficiency or excess of various vital substances, such as calcium, proteins, and vitamins, and how to normalize such substances through balanced diet. Several works [8-15] proposed different recommendation systems related to food. These systems can be categorized as: (a) food recommendation systems [8, 9], (b) menu recommendations [11], (c) diet plan recommendations [12], (d) health recommendations for different diseases like diabetes and cardiovascular [13, 14], and (e) recipe recommendations $[10,15]$. All the aforementioned systems provide recommendations to either some specific disease or balance the diet without considering information about any disease or nutrition deficiency in the body. For instance, in [8], a food recommendation system is proposed for the patients of diabetes. The system recommends various foods for diabetic patients without considering the diabetes level that may fluctuate frequently. Similarly, the authors in [9] do not consider the nutrition factors that have significant importance for a balanced diet recommendation.

Keeping in view the above mentioned facts and figures, it is of critical importance to maintain a balanced intake of food. However, it is quite challenging for a common person to keep track of personal food requirements because of the massive diversity of dietary components and items. A systematic food recommendation system is desired to recommend the appropriate food considering the disease of the person. The major challenge in designing such a system is the handling of greater volumes of data in terms of ingredients, quantity, nutrition facts, people's preferences, and simultaneously taking into consideration a person's pathological reports. The system must be scalable enough to handle recommendation queries from all over the globe. A solution to the aforementioned challenges is the use of cloud computing. Cloud computing is an innovative and emerging platform that enables users to perform on-demand scalability of computing and storage resources [16].

In this article, we present a cloud based food recommendation system called Diet-Right that considers the users' pathological tests results, and recommends a list of optimal food items. To achieve optimal results, we developed an algorithm based on Ant Colony Optimization (ACO). We designed a database of 345 pathological test reports and their normal ranges. A database was created by performing a field survey and collecting the information about pathological reports from different laborities $[17,18,19]$. The collected data was verified by a pathalogist of a hospital. Moreover, a database of 3,400 food items with 26 entries for most common nutrition was taken from the official website of composition of foods integrated dataset 
(CoFID) [20]. Based on the real-time input of user's parameters, the Diet-Right recommends top ranked food items to the user.

The rest of the paper is organized as follows. Section 2 discusses related work. Section 3 presents the system model and recommendation process. Section 4 presents the experimental setup and results, and Section 5 concludes the paper.

\section{Related Work}

Several works have been proposed for different recommendation systems related to diet and food. These systems are used for food recommendations, menu recommendations, diet plan recommendations, health recommendations for specific diseases, and recipe recommendations. Majority of these recommendation systems extract users' preferences from different sources like users ratings [21, 22], recipe choices [23, 24], and browsing history [25, 26, 27]. For instance, in [24], a recipe recommendation system is proposed using social navigation system. The social navigation system extracts users' choices of recipes and in return recommends the recipes. Similarly, in [27], a recipe recommendation system is proposed that is capable of learning similarity measure of recipes using crowd card-sorting. The above mentioned recommendation systems lack in solving a common problem known as cold start problem. All these system must wait for the users to enter enough data for the effective recommendations [28]. Some of the commercial applications like [29, 30] offer users for a quick survey in order to get users preferences in a short time. For instance, the survey used by [29] is specifically designed to match the lifestyle of the user i.e., healthy, sportsman, pregnant, etc. The survey also attempts to avoid various foods which do not match the user's lifestyle. Similarly, a questionnaire is used by [30] through which a user answers different questions about his/her lifestyle, food preferences, nutrient intake, and habits. The system once extracts all the basic information is then able to recommend different meals for daily and weekly basis.

A Food Recommendation System (FRS) [8] is proposed for diabetic patients that used K-mean clustering and Self-Organizing Map for clustering analysis of food. The proposed system recommends the substituted foods according to nutrition and food parameters. However, FRS does not adequately address the disease level issue because the level of diabetes may vary hourly in different situations of the patient and the food recommendations may also vary accordingly. Tags and latent factor are used for android based food recommender system [9]. The system recommends personalized recipe to the user based on tags and ratings provided in user preferences. The proposed system used latent feature vectors and matrix factorization in their algorithm. Prediction accuracy is achieved by use of tags which closely match the recommendations with users' preferences. However, the authors do not consider the nutrition factors in order to balance the diet of the user according to his needs. Content based food recommender system [10] is proposed which recommend food recipes according to the preferences already given by the user. The preferred recipes of the user are fragmented into ingredients which are assigned ratings according to the stored users' preferences. The recipes with the matching ingredient are recommended. The authors do not consider the nutrition factors and the balance in the diet. Moreover, chances of identical recommendation are also present because the preference of the user may not change on daily basis. In [31], knowledge based dietary nutrition recommendation system is proposed for obesity. The recommendations include dietary nutrition and diet menus for individuals using collaborative filtering technique. An application for mobile users is also developed in order to recommend the dietary nutrition 
and menus to the users. Similarly, a food recommender system is proposed in [32] for patients in care facilities. The application is designed for caregivers in the care facilities in order to offer the food according to the patient preferences.

The above mentioned food recommendation systems are specifically dealing with some diseases or related to balance the diet. In case of food recommendation for specific diseases, the systems recommend different foods for patients without knowing the level of disease which may vary in different cases. Similarly, in case of food recommendations to balance the diet, nutrition factors are ignored which are very much important to recommend food and balance diet.

In medical practice, sometimes, pathological tests are required to identify a particular disease. A pathological test report usually indicates deficiency or excess of certain compounds/parameters in human body (e.g., levels of iron, calcium, or RBC count, etc.) which may cause particular disease. In this article, we present a novel food recommendation system specifically dealing with the pathological tests results. Our system considers diseases related to pathological reports, and most common nutrition factors in recommending the food items to the users. For this purpose, we used a database of 345 pathological test reports to categorize various diseases that occur due to the deviation from the normal ranges of compounds/parameters. Moreover, we designed a system that allows users to input values for a specific parameter. Based on the deviations of the input parameter value from the normal ranges, the system generates a diet plan that aims to cover those abnormalities. Furthermore, we used ant colony algorithm to train the system with the values of various parameters' ranges and diseases.

\section{Diet-Right Model}

The main focus of this work is to provide dietary assistance to different people who are suffering from common diseases. The proposed model recommends various foods and nutrition to the people based on their pathological test reports. Every pathological report has some indicators that are calculated based on the nature of the tests. For instance, if a doctor advised a patient to take pathological test of blood, then the common test entries include the values of hemoglobin, red blood cells (RBC), white blood cells (WBC), plasma, and sugar. Normal ranges of the aforementioned indicators are usually given in the test reports. In this way, the patient can identify the abnormalities after comparing with the normal ranges. In our proposed system, a user is provided with the complete list of the test parameters to make selection from. The user inputs the specific values of test report in the selected parameters. We gathered the data of normal ranges for tests including blood (plasma and serum), urine, stool, cerebrospinal fluid (CSF), and gastric and secretion tests. A matrix of 345 entries was constructed. Each individual components of a test (e.g., blood test) have normal ranges with lower and upper bound. The ranges of the same component may differ on the basis of gender, age groups, and fasting or no fasting. Our system is trained on various types of age groups and their respective ranges of parameters. This allows the system to suggest diets as per needs of the users. 


\subsection{Diet-Right Architecture}

In majority of the existing food recommendation systems, centralized architecture is used [8-15]. The main disadvantage of using such systems is scalability, when dealing with the massive amount of data. We propose a cloud based solution to offer the scalability and pervasiveness, where the smart phone users can conveniently access the recommendation system (see Fig. 1).

The model takes the input values as a first step. User enters the demographic data including gender and age as well as the value of the pathological test reports. These values are sent to cloud infrastructure in second step and are compared with the normal ranges that are stored in our database. In the third step, the abnormality level of the pathological test reports is computed. In the next step, the weight assignments and matrix generation process is carried out. In the fifth step, ranks are calculated for each food item and are sorted in descending order. In the sixth step, the user is provided the recommended list of food items.

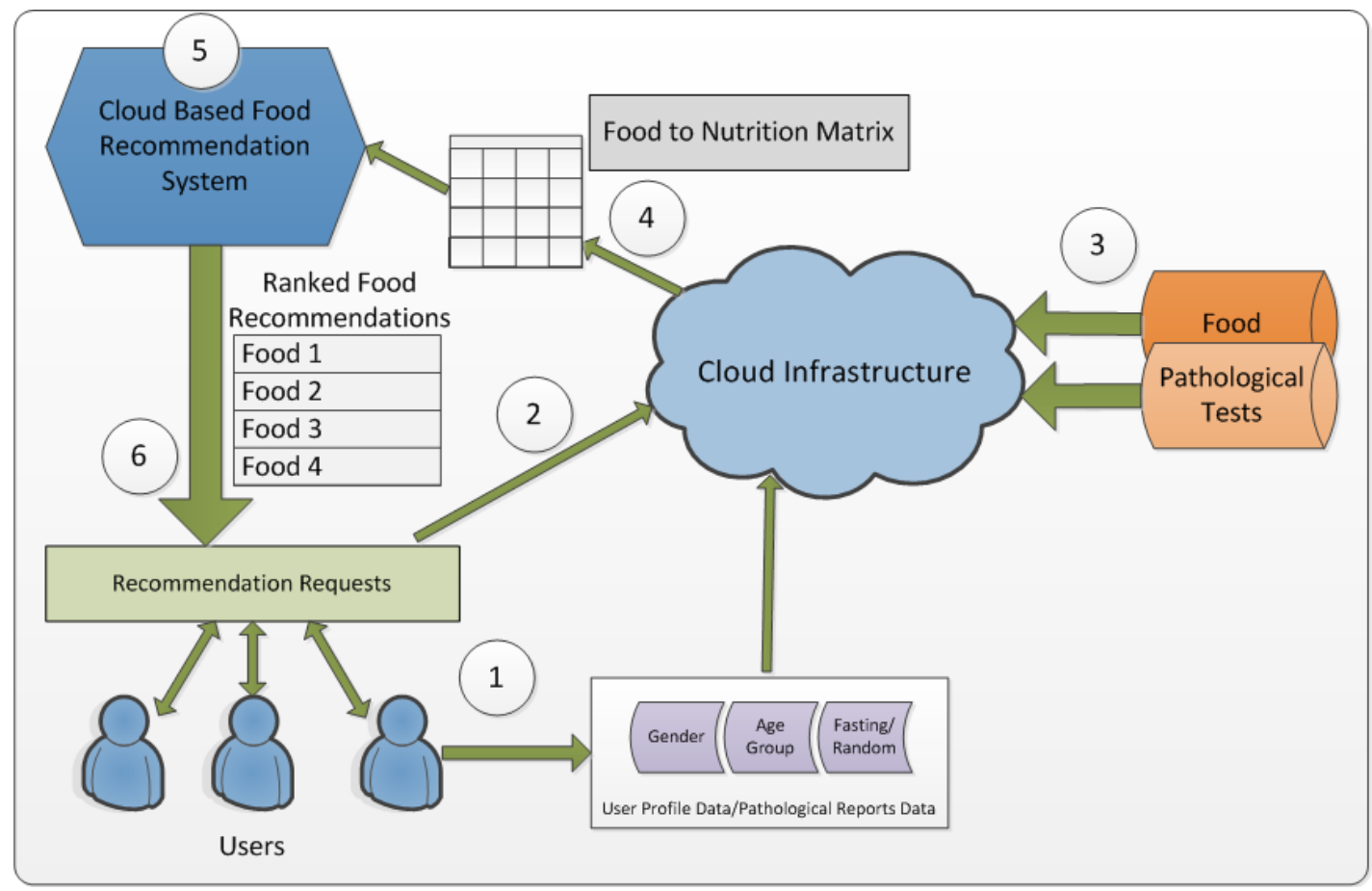

Fig. 1. Architecture of Diet-Right

\subsection{Proposed Algorithm based on Ant Colony Optimization (ACO)}

In this subsection, we present the food recommendation process using variant of ant colony approach on a graph of foods to generate the optimal food set for the users. In Diet-Right, we have used Ant Colony Optimization technique. ACO metahiuristic is a constructive and population based-approach which relies on the social behavior of ants. It is recognized as a most powerful approach for the solution of combinatorial optimization problems [33]. The main steps used in our proposed Algorithm are explained as follows: 
Each food item is placed on nodes and a strongly connected graph is generated as shown in Fig. 2. Each link of graph has associated $\boldsymbol{\eta}$ and $\boldsymbol{\tau}$ values, where $\boldsymbol{\tau}$ is the randomly initialized pheromone, and $\boldsymbol{\eta}$ is the heuristic information initialized as the inverse of squared sum of difference of all the ingredients I. In Equation (1), $\boldsymbol{k}$ represents index for food ingredient, $\boldsymbol{i}$ and $\boldsymbol{j}$ represent $\boldsymbol{i}^{\boldsymbol{t} \boldsymbol{h}}$ and $\boldsymbol{j}^{\boldsymbol{t h}}$ food item, I represents a single ingredient in a certain food item, and $\mathrm{m}$ is the total number of ingredients in a certain food item.

$$
\eta_{i j}=\sum_{k=1}^{m}\left(I_{i k}-I_{j k}\right)^{2}
$$

Where, $\eta$ is used to control exploration and exploitation of ACO and the values of $\eta \in(0,1)$.

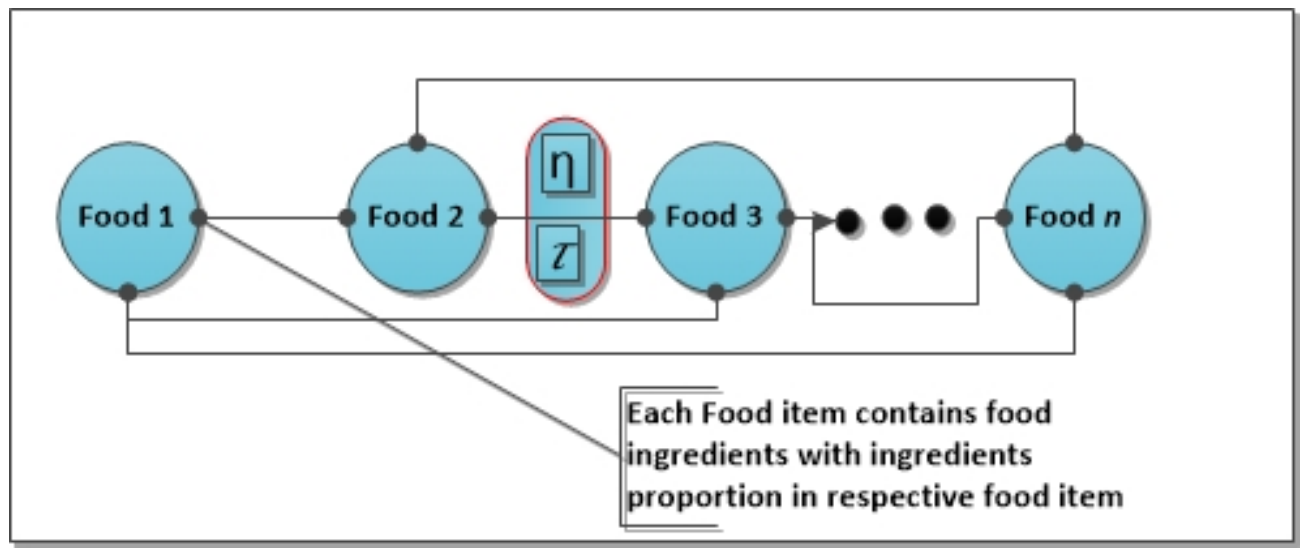

Fig. 2. Graph representation of the problem

After initialization, each ant constructs its local solution by visiting nodes which provide best cost in terms of low error compared to targets. Target vector represents the amount of food ingredients required against the particular disease. Target vector is predefined based on pathological reports, for instance, target vector for user with calcium deficiency may ranges from 9 to 10.5. The different nodes or food items are selected using transition rule which selects a path with highest transition probability. Transition probability is given by Equation (2):

$$
p_{i}^{k}(t)= \begin{cases}\frac{[\tau i(t)]^{\alpha} \times[\eta i(t)]^{\beta}}{\sum_{r \in j^{k}}\left[\tau_{r}(t)\right]^{\alpha} \times\left[\eta_{r}(t)\right]^{\beta}}, & \text { if } i \in j^{k} \\ 0 & \text { Otherwise }\end{cases}
$$

Where, $\boldsymbol{\tau} \boldsymbol{i}(\boldsymbol{t})$ represents the pheromone level at time $\boldsymbol{t}, \boldsymbol{\eta} \boldsymbol{i}(\boldsymbol{t})$ is the heuristic information at time $\boldsymbol{t}$, and $\boldsymbol{\alpha}, \boldsymbol{\beta}$ are the hyper parameters in the model used to weight heuristic information 
and pheromone level (used for fine tuning). Moreover, $\boldsymbol{k}$ represents ant, $\boldsymbol{i}$ represents initial node, $\boldsymbol{j}$ is the target node, $\boldsymbol{r}$ is index of current selected path, and $\boldsymbol{j}^{\boldsymbol{k}}$ represents the solution.

When an ant selects a path among all existing paths (excluding the path in the solution), it updates the pheromone level locally as depicted in Equation (3).

$$
\tau_{i j}^{k}(t+1)=\left\{\begin{array}{cc}
\tau_{i j}^{k}(t) \times \rho+\delta \tau^{k}, & \text { if } i j \in S^{k}(t) \\
(1-\rho) \tau_{i j}^{k}(t) & \text { otherwise }
\end{array}\right.
$$

Where, $\boldsymbol{\tau}_{\boldsymbol{i j}}^{\boldsymbol{k}}(\boldsymbol{t}+\mathbf{1})$ is new pheromone level that is increased by amount $\boldsymbol{\delta} \boldsymbol{\tau}^{\boldsymbol{k}}$, and evaporation is governed by multiplication of pheromone decaying parameter $\boldsymbol{\rho}$. Also, $\boldsymbol{S}$ is the solution of the $\boldsymbol{k}^{\text {th }}$ ant at time $\boldsymbol{t}$.

Each ant provides locally optimized food set based on the nutrition expert recommendations, but here, we are interested in the globally optimized solution. As we are using supervised approach, we use Root Mean Square Error (RMSE) for the selection of globally best solution. To do so, we initialize global solution to EMPTY set and solve for each ant. Initially, solution returned by the first ant is considered as the global solution. Afterwards, when rest of the ants return with a solution, their RMSE is compared with the current global solution replacing the global solution with the solution having minimum RMSE value. For fast convergence of the solution, we update the pheromone level again using the same formula, but the update is only for the path that is globally optimal solution as depicted in Equation (4).

$$
\tau_{i j}(t+1)=\left\{\begin{array}{cc}
\tau_{i j}^{g}(t) \times \rho+\delta \tau^{g}, & \text { if } i j \in S^{g}(t) \\
(1-\rho) \tau_{i j}^{g}(t) & \text { otherwise }
\end{array}\right.
$$

In food selection process, there is a need to select diversified foods to enhance the acceptance of foods among different people. We manage and update the heuristic information in such a way that the diversity among foods is maximized. For heuristic information update, we use Equation (5).

$$
\eta_{i}=\frac{1}{m_{i}} \sum_{k=1}^{n} \gamma\left(S^{k}(t)\right)\left(1+\phi_{i} e^{\frac{-\left|S^{k}(t)\right|}{n}}\right), \text { if } i \in S^{t}(t)
$$

Where, $\boldsymbol{m}$ is the selected number of foods, $\boldsymbol{\phi}_{\boldsymbol{i}}$ is the number of times a food is selected in whole iteration, and $\mathrm{m}, \boldsymbol{\phi}_{\boldsymbol{i}}$ are the parameters used to balance the solution in terms of local and global perspective. The used heuristic information faciliates in the selection of foods with minimal redundancy. Alorithm 1 presents food recommendation using ACO.

\begin{tabular}{l} 
Algorithm 1: Food Recommendation using ACO \\
\hline $\begin{array}{l}\text { Input: Dataset (f, } \mathrm{n}) \mathrm{f} \text { foods, } \mathrm{n} \text { nutrition, and } \mathrm{T}(\mathrm{n}) \text { prescribed } \\
\text { nutrition plan }\end{array}$
\end{tabular}

Output: Selection of Optimized Food Set R based T (n) 
1: Set initial values of the heuristic information $\eta i j, \rho$ and level of pheromone trails $\tau_{i, j}$ randomly and Nants $\leftarrow k$

2: $\mathrm{Sg} \leftarrow \varnothing$

3: repeat

4: $\quad$ Generate and randomly place ants at different nodes of the graph

5: $\quad k \leftarrow 1$

6: $\quad$ for $k \leq$ Nants do

7: $\quad$ Construct Solution $S^{k}$ for each Ant using transition and pheromone update

8: $\quad$ while (all nodes are not visited) do

9: $\quad$ Calculate transition probability using (2)

10: $\quad$ With constraint $S^{k}(t) \neq S^{k}(t+1)$ for $k=1,2,3, \ldots, n$

11: $\quad$ Update pheromone locally $\tau_{i j}^{k}$ using (3)

12: $\quad$ Add $\arg _{\max }\left\{p_{i}^{k}(t)\right\}$ to $S^{k}(\mathrm{t})$

13: $\quad$ end while

14: $\quad$ if $\left(S^{g}=\emptyset\right)$ then

15: $\quad S^{g}(t)=S^{k}(t)$

16:

else if $\left(\sqrt{\left(T-\sum_{i=1}^{\text {size }\left(S^{g}\right)} S_{i}^{g}\right)^{2}} \geq \sqrt{\left(T-\sum_{i=1}^{\text {size }\left(S^{k}\right)} S_{i}^{k}\right)^{2}}\right)$ then

17: $\quad S^{g}(t)=S^{k}(t)$

18: $\quad$ else

19: do nothing

20: $\quad$ end if

21: $\quad$ end for

22: $\quad$ Update pheromone globally $\tau_{i j}^{g}$ using (4)

23: $\quad$ Update heuristic information $n_{i}$ using (5)

24: $\quad E\left(T, S^{g}\right)=\sqrt{\left(T-\sum_{i=1}^{\text {size }\left(S^{g}\right)} S_{i}^{g}\right)^{2}}$

25: until $E\left(T, S^{g}\right) \geq T_{H}$ or maximum iterations reached

26: Return $S^{g}$

\subsection{Ranked List Generation}

If a single item is selected, the foods are recommended based on parameters relevant to the item. For instance, in case of uric acid, purine quantity in the foods is considered. However, if multiple items are selected, then the following equation is used.

$$
T_{w}=\sum_{i=1}^{n} P_{i} W_{i}
$$

Where, $\boldsymbol{T}_{\boldsymbol{w}}$ represents the total weight of the food item, $\boldsymbol{P}_{\boldsymbol{i}}$ represents a single parameter (e.g., purine, carbohydrates, etc.), and $\boldsymbol{W}_{\boldsymbol{i}}$ represents the importance of the food item (value lies between 0 and 1). The weight $\boldsymbol{T}_{\boldsymbol{w}}$ of the food items facilitate to rank the foods based on the selected items. Food items consist of $\boldsymbol{P}_{\boldsymbol{n}}$ nutrition and each pathological test indicates certain 
deficiencies of $\boldsymbol{P}_{\boldsymbol{n}}$ in human body. Therefore, the weights $\boldsymbol{W}_{\boldsymbol{i}}$ are introduced to compensate the tradeoff between different test substances. Weight assignment of different foods is stored in food-to-nutrition matrix where each row of the matrix represents a food plan. There are $m$ number of food plans each having $n$ numbers of food items in every food plan. The matrix generates ranked food plans which are used by the user.

\section{Experimental Setup and Results}

Extensive simulations are conducted to evaluate performance of the proposed system. The experimental setup and results are discussed as follows.

\subsection{Experimental Setup}

The details of the experimental setup and parameters used for evaluation are presented in Table 1.

Table 1. Experimental Setup

\begin{tabular}{|l|l|}
\hline Parameters & Values \\
\hline Total Number of Food Items & 3400 \\
\hline Nutrition for Each Food Item & 26 \\
\hline Total Number of Pathological Test Reports & 345 \\
\hline Number of Ants used for Simulation & $10-120$ \\
\hline Maximum Iterations for each Ant & 200 \\
\hline Simulation Tool & MATLAB \\
\hline Single Node System Configuration & RAM 16 GB, Cores 4 \\
\hline Cloud Configuration & MATLAB Parallel Cloud, Cores 16 \\
\hline
\end{tabular}

\subsection{Results}

We analyzed the behavior of proposed algorithm in terms of time complexity. We observed that increasing number of ants converge the solution to its minimum cost, but practically, it is not feasible to use high number of ants. Moreover, using high number of ants to contract a solution increases the time complexity as shown in Fig. 3. 


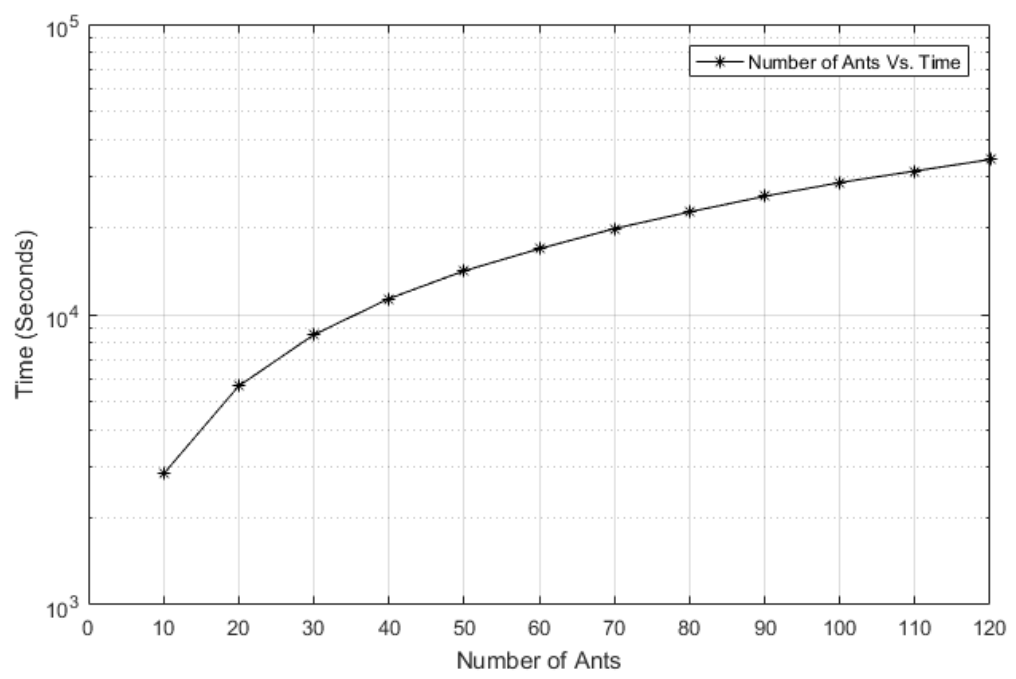

Fig. 3. Tradeoff between numbers of ants to time complexity

To select optimal number of ants for best results irrespective of time complexity, RMSE was estimated. It is observed that 110 ants produce lowest error rate. As our algorithm uses random initialization, it produced varied results. To address this abnormality, we used average of 10 executions with same settings. It can be observed that with increasing number of ants, RMSE is decreasing. Fig. 4 shows average RMSE withrespect to increasing number of ants.

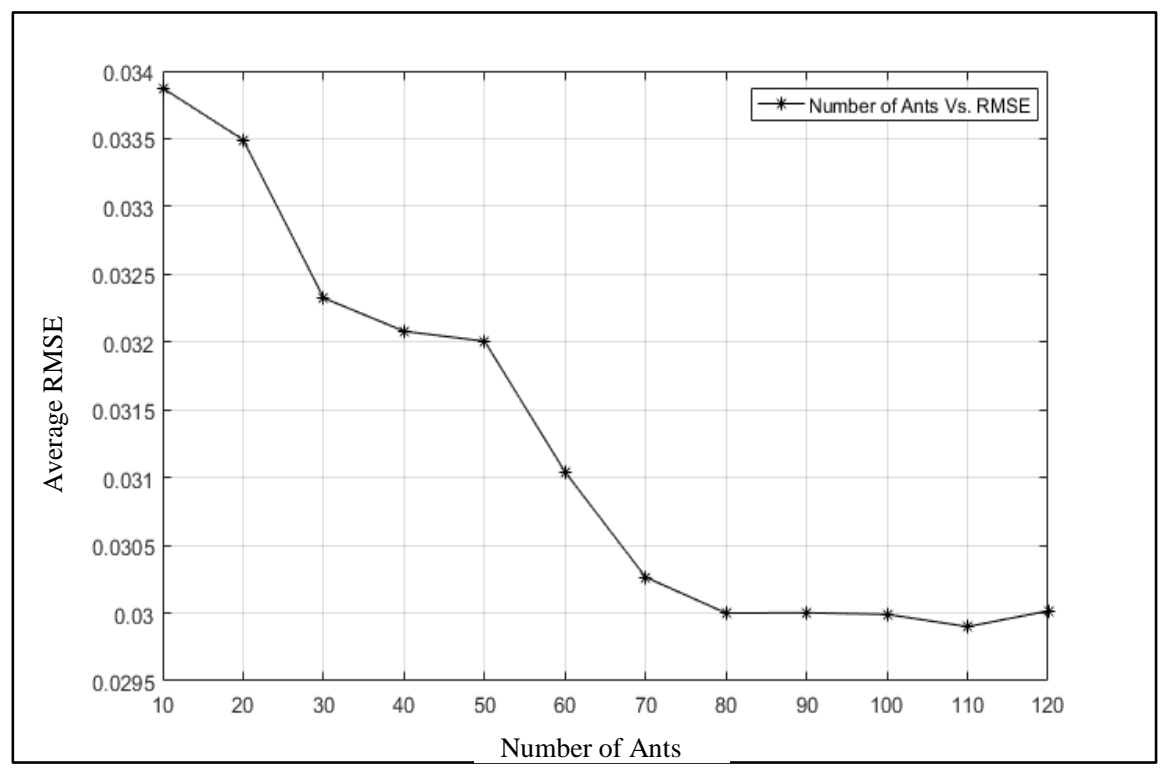

Fig. 4. Tradeoff between number of ants and RMSE

For selection of optimal number of ants, we performed best cost analysis for number of iterations versus number of ants. It is evident in Fig. 5 that in our case, 80 ants provide best results in terms of converence of the solution. 


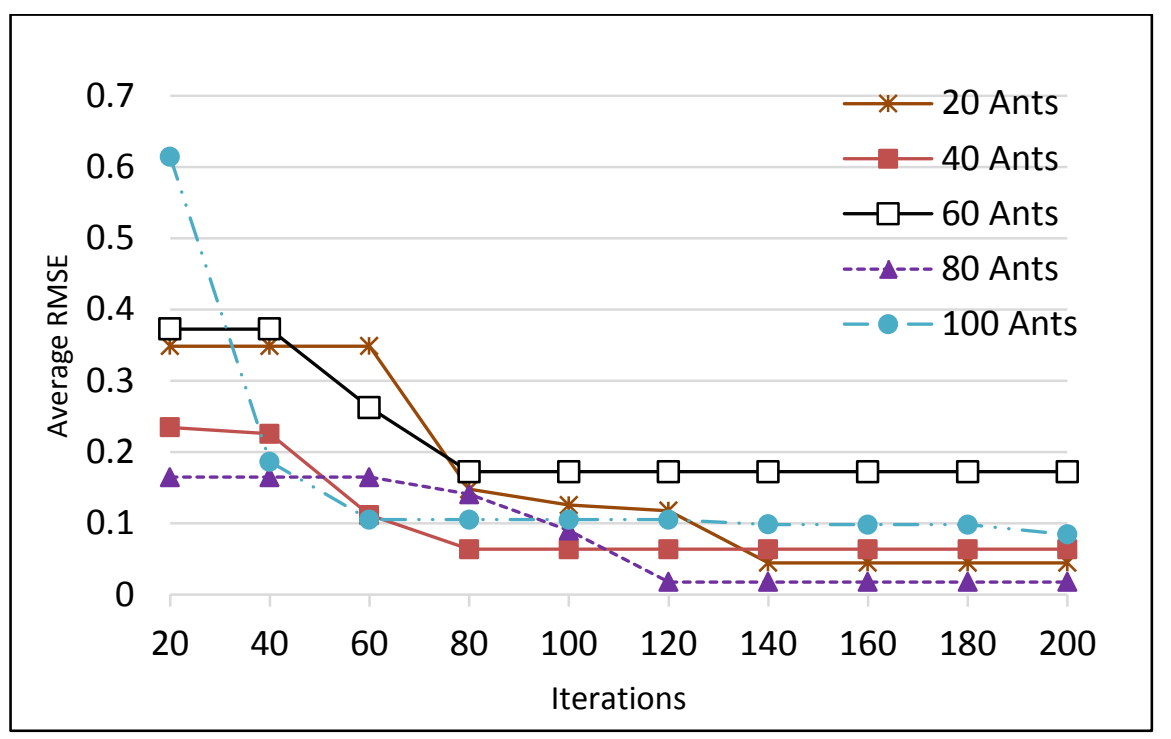

Fig. 5. Cost over Varying No. of Ants and Iterations

Fig. 6 presents the convergence time of different diseases. As shown in the previous result, 80 ants provide the best result in terms of convergence of the solution. Therefore, we have used 80 ants for the convergence time comparison between the most common diseases. The result shows that the convergence time for normal person is higher compared to persons with some disease. On the other hand, the convergence time to recommend foods for a hypertension patient is significantly lower compared to others. The reason for such variance is that the number of foods available for a normal/healthy person is much higher compared to the number of foods that are avilable for a patient.

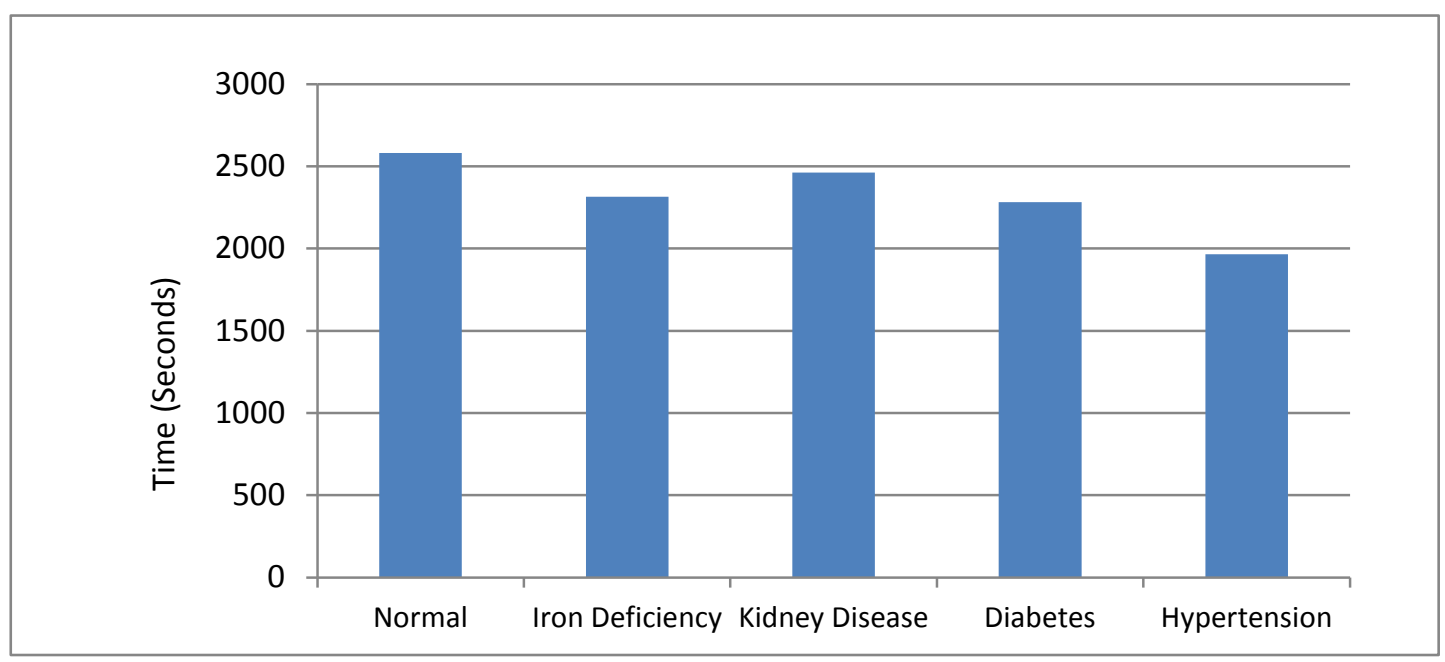

Fig. 6. Convergence Time of Different Diseases 
Fig. 7 illustrates the cost comparison of common diseases. It can be seen that least cost is achieved for hypertension, whereas normal person exhibited highest cost compared to others. This shows that the dataset used in this study is more suitable for certain diseases, such as hypertension.

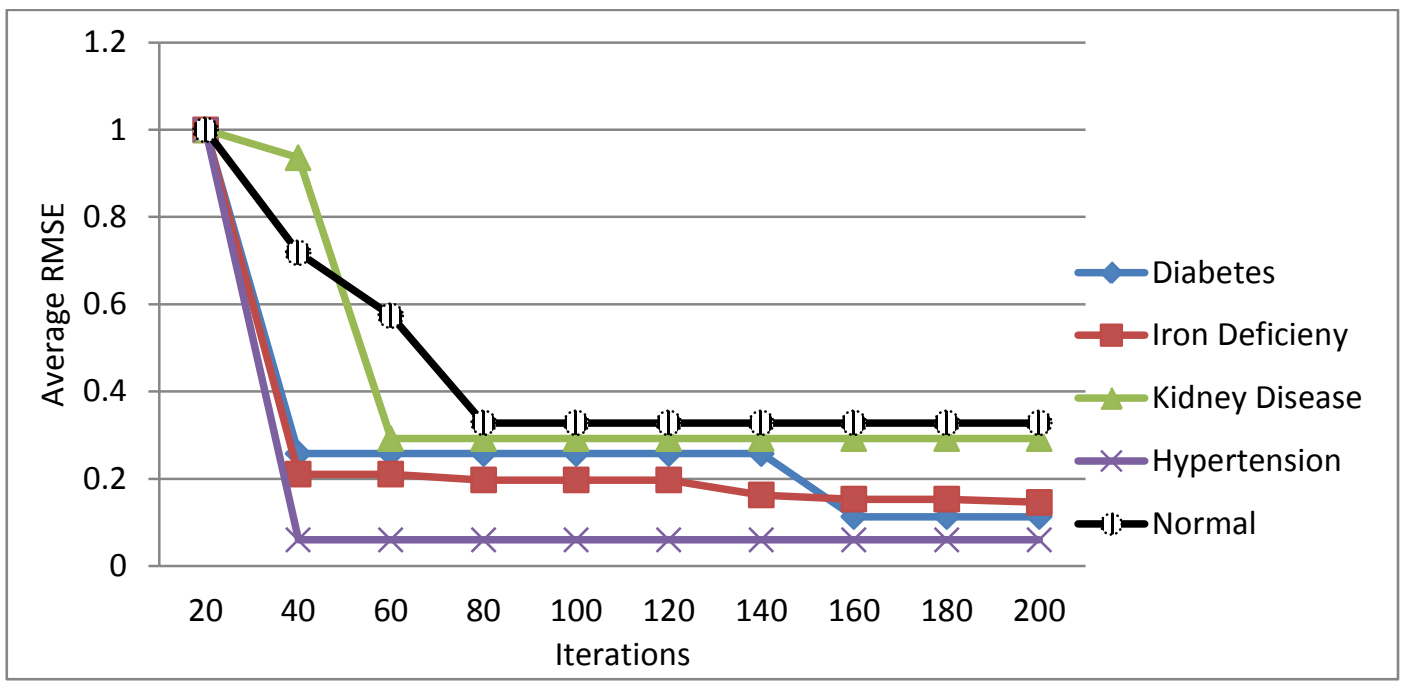

Fig. 7. Cost Comparisons of Diseases

Fig. 8 depicts the accuracy of recommendations relative to number of ants. The result shows that the highest accuracy is achieved with 110 ants. It is quite evident that when we increase the number of ants, the accuracy is also increased. Moreover, it is observed that the accuracy remains constant between 80 to 100 ants.

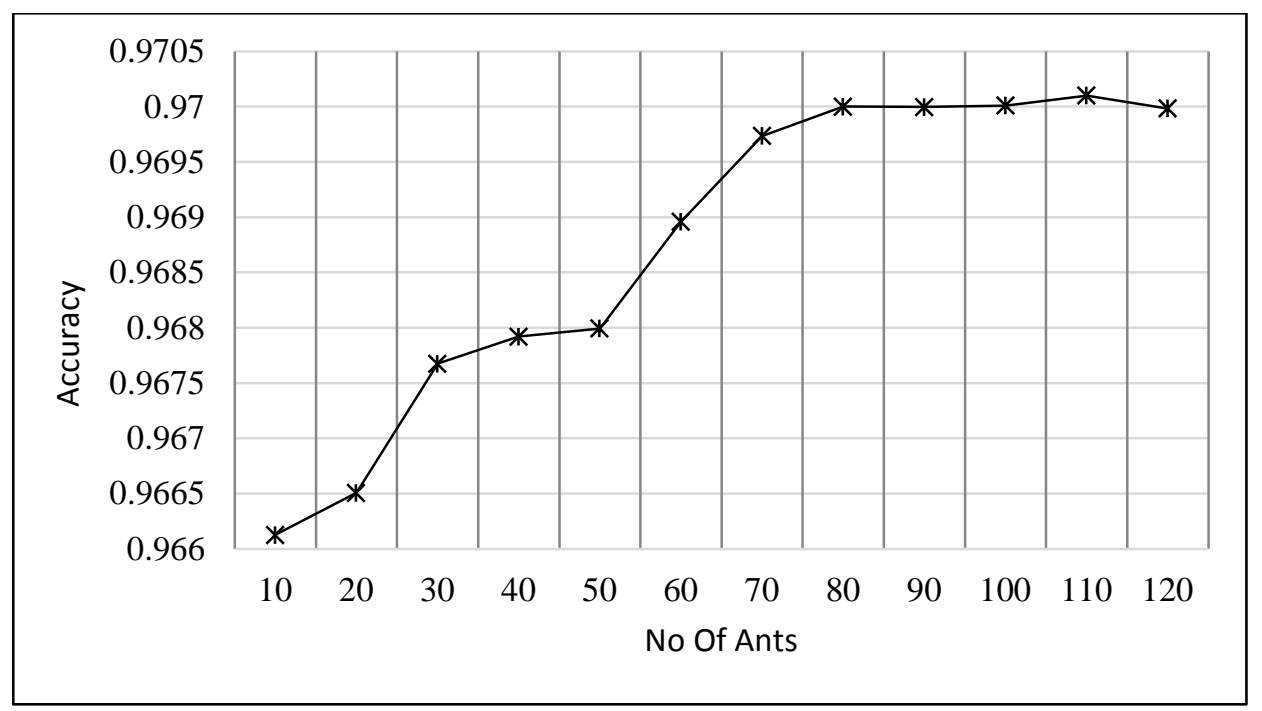

Fig. 8. Accuracy of Recommendations 
Fig. 9 depicts the convergence time of single node and cloud-based execution. For this experiment, we executed our algorithm using Matlab's cloud framework [34]. It is evident from the result that the convergence time is significantly reduced with cloud-based execution. It is noteworthy that the convergence time of cloud based execution is approximately 12 times lower (on average) compared to single node execution.

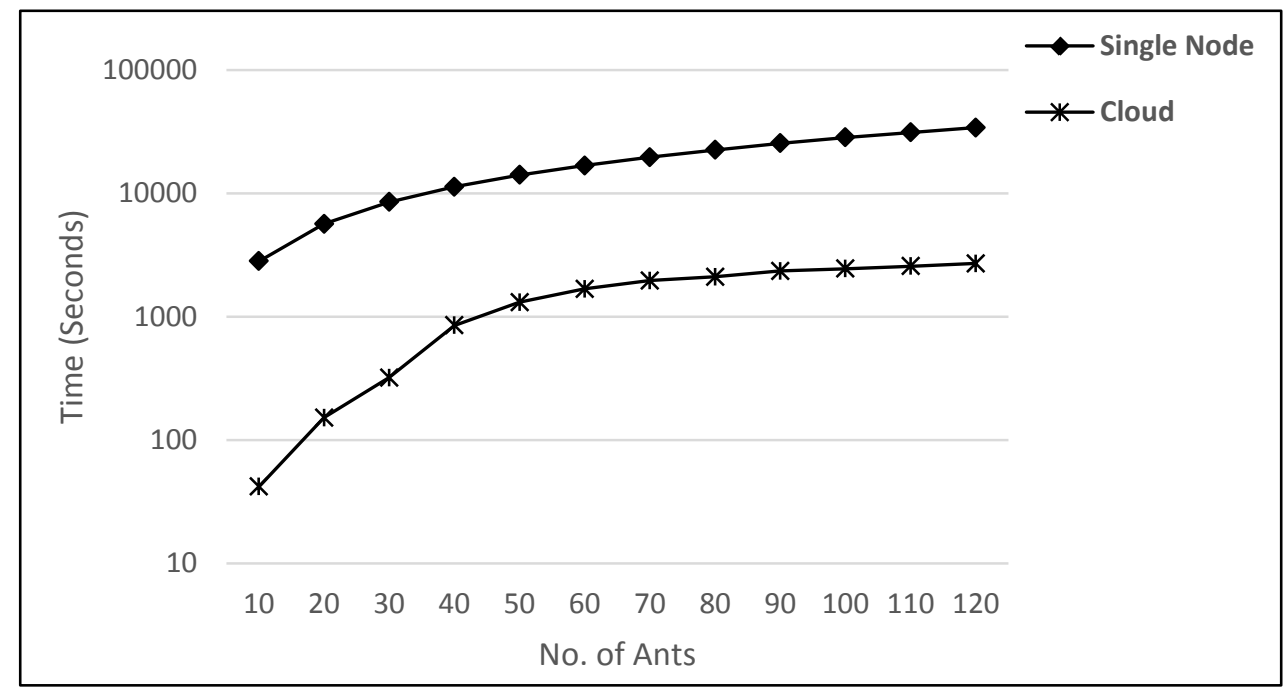

Fig. 9. Convergence Time of Single Node and Cloud based Execution

\section{Conclusions}

In this paper, we presented a cloud based food recommendation system called Diet-Right. Based on user input, it recommends a list of optimal food items using an ACO model. Diet-Right manages and updates the heuristic information in such a way that the diversity among foods is maximized. Extensive expermentation was performed to check the cost, accuracy, convergence time, and performance gain. As a future research, we will focus on the recommendations breakdown for different timings of the day, such as breakfast, lunch, and dinner. Moreover, we will consider the amount of nutrition in different food items as per timing and daily needs of the patients. Furthermore, group food recommendation for family/friends is another interesting research area that can be explored.

\section{References:}

[1] Oh, Yoosoo, Ahyoung Choi, and Woontack Woo, "u-BabSang: a context-aware food recommendation system," The Journal of Supercomputing 54, no. 1, 61-81, 2010. Article (CrossRef Link)

[2] A global agenda for combating malnutrition: progress report. Geneva, World Health Organization, 2000. Article (CrossRef Link)

[3] Childhood nutrition and progress in implementing the International Code of Marketing of Breast-milk Substitutes. Geneva, World Health Organization, 2002. Article (CrossRef Link)

[4] WHO, Joint, and FAO Expert Consultation, "Diet, nutrition and the prevention of chronic diseases," World Health Organ Tech Rep Ser 916, no. i-viii, 2003. Article (CrossRef Link) 
[5] WHO/UNICEF. Global prevalence of vitamin A deficiency, MDIS Working Paper No. 2. Geneva, World Health Organization, 1995. Article (CrossRef Link)

[6] WHO/UNICEF/United Nations University. Iron deficiency anaemia assessment, prevention and control: a guide for programme managers, Geneva, World Health Organization, 2001. Article (CrossRef Link)

[7] WHO/UNICEF/International Council for the Control of Iodine Deficiency Disorders, Progress towards the elimination of iodine deficiency disorders (IDD). Geneva, World Health Organization, 1999. Article (CrossRef Link)

[8] Phanich, M., Pholkul, P., \& Phimoltares, S., "Food recommendation system using clustering analysis for diabetic patients," in Proc. of International Conference on Information Science and Applications, pp. 1-8, IEEE, April 2010. Article (CrossRef Link)

[9] Ge, M., Elahi, M., Fernaández-Tobías, I., Ricci, F., \& Massimo, D., "Using tags and latent factors in a food recommender system," in Proc. of the 5th International Conference on Digital Health, pp. 105-112, ACM., May 2015. Article (CrossRef Link)

[10] Freyne, J., \& Berkovsky, S., "Evaluating recommender systems for supportive technologies," User Modeling and Adaptation for Daily Routines, pp. 195-217, Springer London, 2013. Article (CrossRef Link)

[11] Runo, M., “FooDroid: A Food Recommendation App for University Canteens,” Unpublished semester thesis, Swiss Federal Institute of Theology, Zurich, 2011. Article (CrossRef Link)

[12] Su, C. J., Chen, Y. A., \& Chih, C. W., "Personalized ubiquitous diet plan service based on ontology and web services," International Journal of Information and Education Technology, 3(5), 522, 2013. Article (CrossRef Link)

[13] Evert, A. B., Boucher, J. L., Cypress, M., Dunbar, S. A., Franz, M. J., Mayer-Davis, E. J., \& Yancy, W. S., "Nutrition therapy recommendations for the management of adults with diabetes,” Diabetes care, 37(Supplement 1), S120-S143, 2014. Article (CrossRef Link)

[14] LeFevre, M. L., "Behavioral counseling to promote a healthful diet and physical activity for cardiovascular disease prevention in adults with cardiovascular risk factors: US Preventive Services Task Force Recommendation Statement, Annals of internal medicine, 161(8), 587-593, 2014. Article (CrossRef Link)

[15] Teng, C. Y., Lin, Y. R., \& Adamic, L. A., "Recipe recommendation using ingredient networks," in Proc. of the 4th Annual ACM Web Science Conference, pp. 298-307, ACM, June 2012. Article (CrossRef Link)

[16] Rittinghouse, J. W., \& Ransome, J. F., “Cloud computing: implementation, management, and security,” CRC press, 2016. Article (CrossRef Link)

[17] Shaukat Khanum Laboratory,_https://shaukatkhanum.org.pk/, accessed December 13, 2016.

[18] Agha Khan Laboratory, https://www.aku.edu/labreports/Pages/default.aspx, accessed December 13, 2016

[19] Healthways Laboratory, http://www.healthwayslabs.net, accessed December 13, 2016.

[20] COFIDS Dataset, https://www.gov.uk/government/publications/composition-of-foodsintegrated-dataset-cofid, accessed September 03, 2016.

[21] Forbes, P., \& Zhu, M., "Content-boosted matrix factorization for recommender systems: experiments with recipe recommendation," in Proc. of the fifth ACM conference on Recommender systems, pp. 261-264, ACM, October 2011. Article (CrossRef Link)

[22] Freyne, J., \& Berkovsky, S., "Intelligent food planning: personalized recipe recommendation,” in Proc. of the 15th international conference on Intelligent user interfaces, pp. 321-324, ACM, February 2010. Article (CrossRef Link)

[23] Geleijnse, G., Nachtigall, P., van Kaam, P., \& Wijgergangs, L., “A personalized recipe advice system to promote healthful choices," in Proc. of the 16th international conference on Intelligent user interfaces, pp. 437-438, ACM, February 2011. Article (CrossRef Link)

[24] Svensson, M., Höök, K., \& Cöster, R., "Designing and evaluating kalas: A social navigation system for food recipes,” ACM Transactions on Computer-Human Interaction (TOCHI), 12(3), 374-400, 2005. Article (CrossRef Link)

[25] Nutrino, URL: https://nutrino.co/, accessed October 10, 2016. 
[26] Ueda, M., Asanuma, S., Miyawaki, Y., \& Nakajima, S. "Recipe recommendation method by considering the user's preference and ingredient quantity of target recipe," in Proc. of the International MultiConference of Engineers and Computer Scientists, Vol. 1, 2014.

Article (CrossRef Link)

[27] Van Pinxteren, Y., Geleijnse, G., \& Kamsteeg, P., "Deriving a recipe similarity measure for recommending healthful meals," in Proc. of the 16th international conference on Intelligent user interfaces, pp. 105-114, ACM, February 2011. Article (CrossRef Link)

[28] Yang, L., Hsieh, C. K., Yang, H., Dell, N., Belongie, S., \& Estrin, D., "Yum-me: Personalized Healthy Meal Recommender System,” arXiv preprint arXiv, 1605.07722, 2016. Article (CrossRef Link)

[29] ShopWell, http://www.shopwell.com/, accessed October 10. 2016.

[30] Yummly, http://developer.yummly.com, accessed October 10. 2016.

[31] Jung, H., \& Chung, K., "Knowledge-based dietary nutrition recommendation for obese management,” Information Technology and Management, 17(1), 29-42, 2016. Article (CrossRef Link)

[32] De Pessemier, T., Dooms, S., \& Martens, L., “A food recommender for patients in a care facility, in Proc. of the 7th ACM conference on Recommender systems, pp. 209-212, ACM, October 2013. Article (CrossRef Link)

[33] DeléVacq, A., Delisle, P., Gravel, M., \& Krajecki, M., "Parallel ant colony optimization on graphics processing units,” Journal of Parallel and Distributed Computing, 73(1), 52-61. 2013. Article (CrossRef Link)

[34] Matlab Parallel Cloud, http://www.mathworks.com/products/parallel-computing/matlabparallel-cloud/, accessed October 10. 2016.

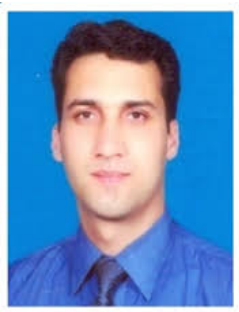

Faisal Rehman received his M.S. in Computer Science from COMSATS Institute of Information Technology, Abbottabad, Pakistan in 2010. He is currently a Ph.D. candidate in the Department of Computer Science, COMSATS Institute of Information Technology, Abbottabad, Pakistan. His research interests include social networks, recommendation systems, and wireless networks. Contact him at frehman@ciit.net.pk

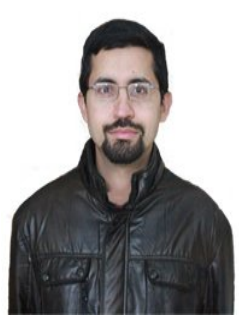

Osman Khalid completed his Ph.D. in 2014 at the North Dakota State University, Fargo, USA. Previously, he obtained degree of Masters in Computer Engineering from Center for Advanced Studies in Engineering (CASE), Islamabad - Pakistan. His area of research includes opportunistic networks, recommendation systems, and trust and reputation systems. He is Assistant Professor in COMSATS Institute of Information Technology, Abbottabad, Pakistan. Contact him at osman@ciit.net.pk 


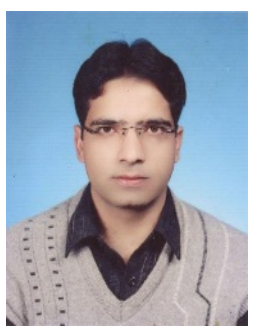

Nuhman ul Haq received his_M.S. in Computer Science from COMSATS Institute of Information Technology, Abbottabad, Pakistan in 2011. He is currently a Ph.D. candidate in the Department of Computer Science, COMSATS Institute of Information Technology, Abbottabad, Pakistan. His research interests include Artificial Intelligence, Image Processing, Neuroscience, and Computational Intelligence. Contact him at nuhman@ciit.net.pk

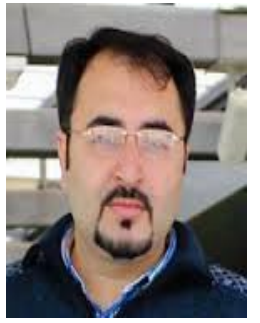

Atta ur Rehman Khan is an Assistant Professor at College of Computer and Information Sciences, King Saud Univeristy. He has completed renowned projects, published research articles in reputed journals/conferences, and edited/co-authored multiple books. Currently, he is an Associate Editor of IEEE Access, Springer Journal of Cluster Computing, Associate Technical Editor of IEEE Communications Magazine, Editor of Elsevier Journal of Network and Computer Applications, IEEE SDN Newsletter, KSII Transactions on Internet and Information Systems, SpringerOpen Human-centric Computing and Information Sciences, SpringerPlus, and Ad hoc \& Sensor Wireless Networks journal, member of IEEE R\&D committee for Smart Grids, IEEE Smart Grids Education Committee, IEEE Smart Grids publications committee, IEEE Smart Grids Operations Committee, Amazon Web Services (AWS) Educate program, NVIDIA GPU Educators Program, Track Chair/ Technical Program Committee (TPC) member of over 50 international conferences, and Higher Education Commission (Pakistan) approved PhD supervisor. He also serves as a domain expert for multiple international research funding bodies, namely European Commission and CONICYT National Commission for Scientific and Technological Research, Santiago, Chile. Dr. Khan has received multiple awards, scholarships, and research grants. His areas of research interest include mobile computing, cloud computing, big data analytics, ad hoc networks, IoT, distributed systems, and security. For more updated information, visit his website at www.attaurrehman.com or contact him at dr@attaurrehman.com

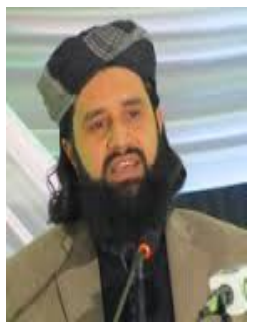

Kashif Bilal is an Assistant Professor at COMSATS Institute of Information Technology Abbottabad, Pakistan. He completed his Ph.D. in 2014 at the North Dakota State University, Fargo, USA. Currently, he is doing post doctrate from Qatar University. His research interests include energy efficient high speed networks and robustness.

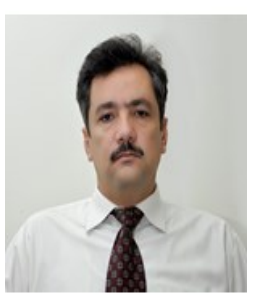

Sajjad A. Madani works at COMSATS Institute of Information Technology (CIIT), Pakistan as Associate Professor. From 2005 to 2008, he worked at Institute of Computer Technology, Vienna University of Technology as a guest researcher where he completed his $\mathrm{PhD}$ studies. He has over 13 years of research and teaching experience at various positions. His areas of interest include wireless networks, cloud computing, social networks, and data center networks. He has published more than 60 papers in peer reviewed journals and conferences. He is actively involved in the organization of different conferences including International Conference on Frontiers of Information Technology, Vice Chancellors Forum at Islamabad, and Pak-China Business Forum. He is senior member IEEE and member Pakistan Engineering Council. Contact him at madani@ciit.net.pk 